Unfallchirurg 2008 $1111: 1033-1036$ DOI 10.1007/s00113-008-1467-z

Online publiziert: 20. August 2008

(c) Springer Medizin Verlag 2008

\section{Redaktion}

W. Mutschler, München

\author{
M. Janke ${ }^{1,2} \cdot$ C. Warzecha1, 2 P.M. Ballmer ${ }^{1}$ \\ 1 Spital STS AG, Thun \\ ${ }^{2}$ Inselspital, Bern
}

\title{
Traumatische chronische Instabilität des proximalen Tibiofibulargelenkes
}

\section{Anamnese und Diagnostik}

Ein 38-jähriger, sportlich aktiver Patient stellt sich $1 \frac{1}{2}$ Jahre nach einer Kniedistorsion beim Skifahren und Konsultation mehrerer Ärzte mit Ausschluss einer ligamentären/meniskalen Verletzung zur Beurteilung chronisch-posterolateraler Kniegelenkschmerzen vor. In der klinischen Untersuchung imponiert ein schmerzhaft um 4-5 $\mathrm{mm}$ nach ventral luxierbares Fibulaköpfchen, bei einem ansonsten unauffälligen Kniegelenkbefund und stabilem kontralateralen proximalen Tibiofibulargelenk (PTFG). Das nach Unfall durchgeführte MRT wurde von mehreren unabhängigen Radiologen und Orthopäden als unauffällig beurteilt.

Nach 6-monatigem konservativem Therapieversuch persistieren bei sportlichen Belastungen deutliche Schmerzen und die ventrale Instabilität nimmt auf 7$8 \mathrm{~mm} \mathrm{zu}$, so dass die Indikation zur operativen Stabilisierung gestellt wird.

\section{Operative Versorgung und Nachbehandlung}

In der von Giachino [4] beschriebenen Technik werden die distale Bizepsfemorissehne, das PTFG und der N. peronaeus dargestellt. Die anterolaterale Luxierbarkeit um 7-8 mm kann bestätigt werden. Unter Belassung der distalen Insertion am Fibulaköpfchen wird die dorsale Hälfte der Bizepsfemorissehne (BFS) proximal abgelöst und der ca. $7 \mathrm{~cm}$ lan- ge Sehnenstreifen am Ende mittels einer Kletternaht mit nicht resorbierbaren Fäden (Fiber Wire ${ }^{\circledast}$ ) angeschlungen (- Abb. 1).

Auf Höhe des Tibiofibulargelenkes wird ein 6-mm-Bohrloch von ventrolateral nach dorsomedial durch die laterale Tibiametaphyse gebohrt. Der Bizepssehnenstreifen wird dorsal in das Bohrloch eingezogen und bei reponiertem PTFG unter Zug ventral über dem Fibulaköpfchen mit den Fäden verknüpft, was zu stabilen Verhältnisse im PTFG führt (- Abb. 2).

Postoperativ erfolgte für 4 Wochen eine Teilbelastung mit $15 \mathrm{~kg}$. Bei beschwerdefreiem Patienten wurde anschließend zur Vollbelastung übergegangen und mit leichter sportlicher Betätigung (Fahrradfahren, Schwimmen) begonnen. Nach 2 Monaten konnte bei freier Kniegelenkbeweglichkeit und klinisch stabilem Tibiofibulargelenk die volle Sportfähigkeit freigegeben werden; 6 Monate nach Operation fand sich eine beschwerdefreie uneingeschränkte Sportfähigkeit bei stabilem PTFG.

\section{Diskussion}

Das PTFG ist ein stabiles Gelenk zwischen lateralem Tibiakondylus und Fibulaköpfchen, welches Torsionsstress aus dem Sprunggelenk aufnehmen muss

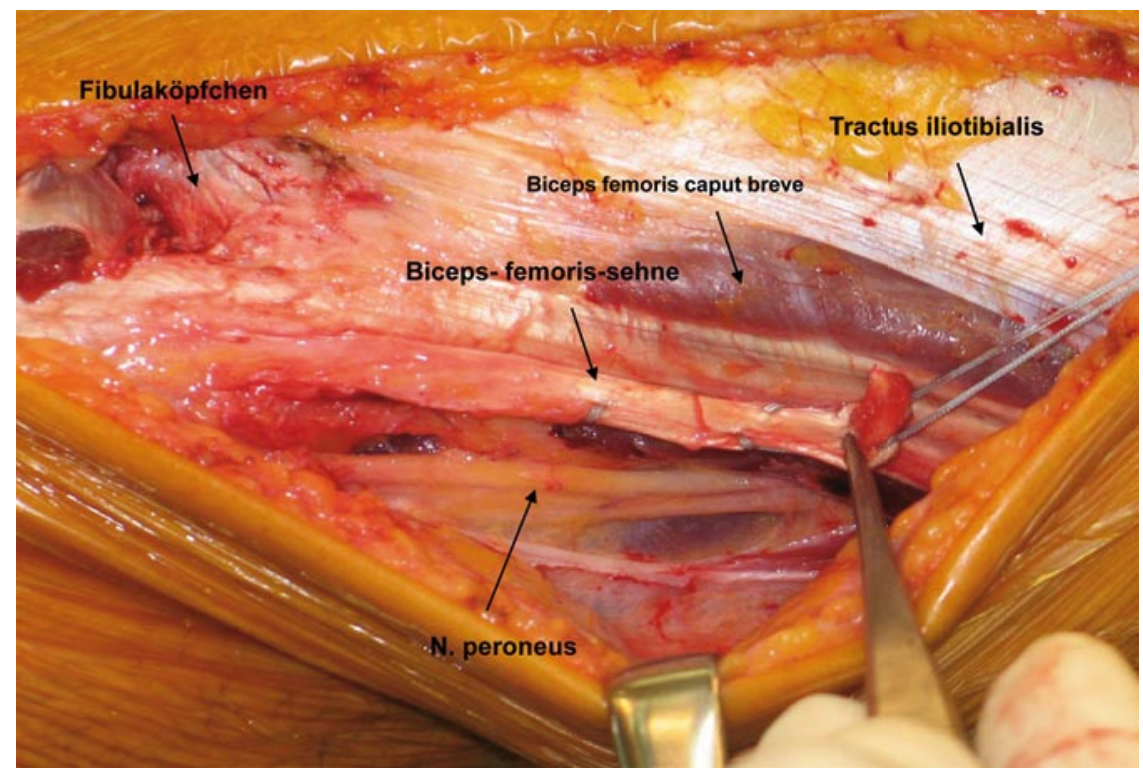

Abb. $1 \Delta$ Angeschlungener dorsaler Streifen der Bizepsfemorissehne 

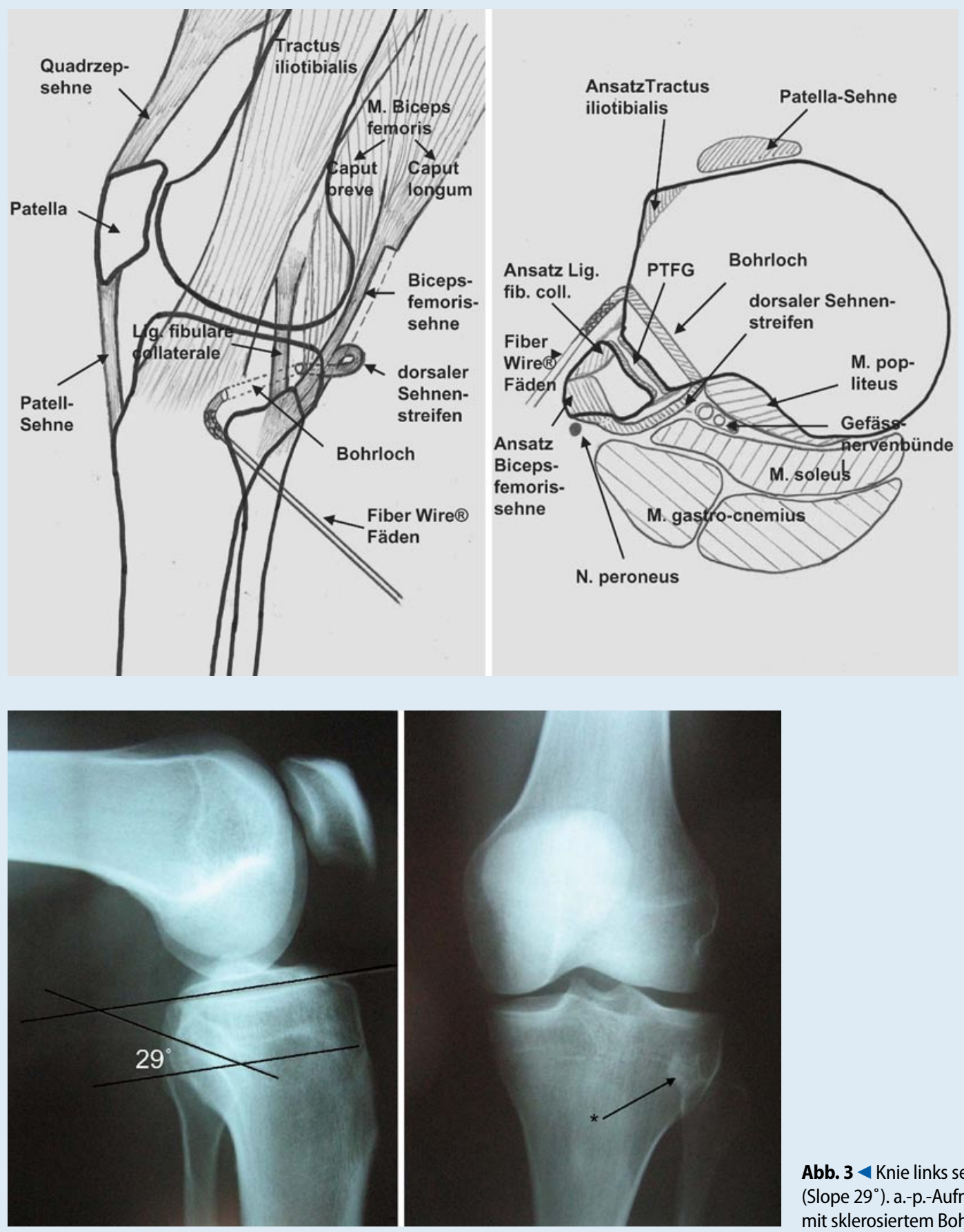

Abb. $3<$ Knie links seitlich:,_Steil/schräg-Typ“ (Slope $29^{\circ}$ ). a.-p.-Aufnahme: postoperatives Bild mit sklerosiertem Bohrkanal $(*)$

$[7,10]$. Die Stabilität wird nach ventral durch die kräftige Gelenkkapsel, das anteriore tibio-fibulare Band und die BFS, nach dorsal durch das posteriore proximale tibiofibulare Band und nach kranial durch das laterale kollaterale Ligament (LCL) gewährleistet $[2,7,10]$. BFS und LCL sind in o- bis 30-Flexion straff, entspannen sich bei zunehmender Flexion, wodurch die proximale Fibula nach ventral gleiten und daraus eine erhöhte
Verletzungsanfälligkeit resultieren kann [7].

Einen weiteren Risikofaktor, eine Instabilität zu entwickeln, stellt der Slope des PTFG dar. Man unterscheidet den häufigeren, „Steil/schräg-Typ” (Slope $>$ 20/straffe kleine Gelenksfläche/geringe Rotationsfähigkeit) und den selteneren "horizontalen“ Typ (Slope <20/große Gelenkfläche/große Rotationsfähigkeit). Die beim „Steiltyp“ bei forcierter Außenro-
Abb. $2<$ Operationsschema mit dorsalem Streifen der Bizepsfemorissehne seitlich und transversal 
Ski- und diversen Ballsportarten), aber auch idiopatisch und selten bilateral auftreten kann $[1,7,8,10]$. Sie kann leicht mit einer Meniskus- oder Seitenbandverletzung, posterolateralen Instabilität oder Läsionen des Tractus iliotibialis und der $B F S$ verwechselt werden. Die im täglichen Leben meist beschwerdefreien Patienten klagen über posterolaterale Kniegelenkschmerzen, evtl. mit schnappenden Sensationen, Blockadegefühl oder Givingway-Episoden.

Bei atraumatischen Subluxationen können durch direkten Druck auf das Fibulaköpfchen die posterolateralen Knieschmerzen verstärkt werden. Die meist weiblichen, jugendlichen Patienten werden häufig im Verlauf des Wachstums asymptomatisch [7].

Bei traumatischer Dislokation des PTFG können die Patienten das Knie nicht belasten, klagen über posterolaterale Schwellung und Schmerzen im Bereich der proximalen Fibula sowie dem Verlauf der BFS, welche sich durch Dorsalflexion des OSG und Extension des Kniegelenkes verstärken lassen. Bei Polytraumapatienten wird die Verletzung häufig übersehen [7].

Bei der klinischen Untersuchung ist v. a. der Seitenvergleich einer schmerzhaften anteroposterioren Instabilität des PTFG wegweisend. Diese lässt sich am besten mit zwischen Daumen und Zeigerfinger gefasstem Fibulaköpfchen bei um $90^{\circ}$ flektiertem Knie und entspanntem LCL/BFS untersuchen. Weiter kann beim liegenden Patienten bei um $90^{\circ}$ flektiertem Knie mittels Innenrotation des Unterschenkels eine Subluxation des Fibulaköpfchens ausgelöst werden (Radulecu-Zeichen; $[7,9,10])$.

Differenzialdiagnostisch ist eine vermehrte dorsolaterale Aufklappbarkeit des Kniegelenkes sowie eine erhöhte Laxizität des normalerweise in Extension stabilen PTFG immer verdächtig auf eine zusätzliche Verletzung des LCL und der dorsolateralen Bandstrukturen. Dies muss wie eine Tendinitis der BFS und Popliteussehne oder eine Meniskusläsion klinisch und ggf. mit bildgebenden Verfahren ausgeschlossen werden (MRT; $[5,7])$.

Da das PTFG nicht mit dem Kniegelenk kommuniziert, sollte ein Infiltrati- onstest des PTFG bei einer rein hierauf begrenzten Pathologie im Gegensatz zu Verletzung von Kniebinnenstrukturen zu einer Beschwerdebesserung führen. Allerdings kann es bei zerrissenen Kapselstrukturen durch Diffusion zu einer Ergebnisverfälschung kommen. In der neueren $\mathrm{Li}$ teratur findet eine Infiltration des PTFG keine Erwähnung und wurde auch in unserem Fall nicht durchgeführt. Als radiologische Standartdiagnostik gelten streng a.-p./seitlich angefertigte konventionelle Röntgenaufnahmen des Kniegelenks, ggf. auch im Seitenvergleich. In der seitlichen Aufnahme sollte eine den posteromedialsten Teil des lateralen Tibiaplateau definierende Linie, welche von der lateralen Eminentia entlang der lateralen Tibiakante nach distal zieht, durch den Mittelpunkt des Fibulaköpfchens verlaufen. Das Fibulaköpfchen liegt bei einer anterioren bzw. posterioren Dislokation vor bzw. hinter dieser Linie [7].

Eine genaue Millimeterangabe bezüglich pathologischer Werte oder Angaben zu Stressaufnahmen unter Durchleuchtung sind in der Literatur nicht zu finden und waren in unserem Fall nicht zur Diagnosestellung notwendig. Aufgrund der außerordentlich großen morphologischen Variabilität [2] ist der Seitenvergleich hilfreicher als absolute Wertangaben. Bei unklarem Befund empfehlen sich axiale Schichten des PTFG im CT oder MRT zur exakten Darstellung der Verletzung $[1,7]$. In jedem Fall bedarf es einer spezifischen Fragestellung, da ein normales MRT des Kniegelenkes das PTFG nur angeschnitten zeigt und diagnostisch nicht verwertbar ist.

Die Klassifikation erfolgt in 4 Typen (• Tab. $1 ;[5,7]$ ).

Die Therapie ist zunächst konservativ mit muskulärer Kräftigung der Hamstring- und Gastrognemikusmuskulatur, Taping, ggf. Ruhigstellung für $2-3$ Wochen und Umstellung der sportlichen Aktivitäten sowie des Trainings unter Vermeidung einer Hyperflexion des Kniegelenkes $[7,8]$.

Bei ausbleibender Besserung nach 6 Monaten muss eine operative Therapie erwogen werden [9]. Unbehandelt kann es zu sekundärer Arthrose im Tibiofibulargelenk kommen $[7,10]$.
Unfallchirurg 2008 $\cdot 111: 1033-1036$

DOI 10.1007/s00113-008-1467-z

(C) Springer Medizin Verlag 2008

M. Janke · C. Warzecha · P.M. Ballmer Traumatische chronische Instabilität des proximalen Tibiofibulargelenkes

\section{Zusammenfassung}

Die seltene chronische Instabilität des proximalen Tibiofibulargelenkes kann traumatisch oder idiopatisch auftreten und im Verlauf zur sekundären Arthrose führen. Nach primär konservativer Therapie sollte bei persistierenden Beschwerden nach 6 Monaten eine operative Therapie in Betracht gezogen werden. Wir präsentieren den Fall einer traumatischen Instabilität, die Rekonstruktion der Kapsel-Band-Strukturen mit der dorsalen Hälfte der Bizepsfemorissehne und anschließender beschwerdefreier Sportfähigkeit.

\section{Schlüsselwörter}

Instabilität · Proximales Tibiofibulargelenk . Traumatisch · Rekonstruktion · Bizepsfemorissehne

Traumatic chronic instability of the proximal tibiofibular joint

Abstract

The rare condition of chronic instability of the proximal tibiofibular joint can be of traumatic or idiopathic origin and can lead to secondary arthritis. After conservative treatment for 6 months and persistent pain, operative treatment should be considered. We present a case of traumatic instability, ligament reconstruction with a part of the biceps femoris tendon, and postoperative return to full and painless sport activities.

\section{Keywords}

Instability - Proximal tibiofibular joint . Traumatic · Reconstruction · Biceps femoris tendon 


\begin{tabular}{|c|c|c|c|}
\hline Typ 1 & Subluxation & $\begin{array}{l}\text { Anterior-posteriore Hyper- } \\
\text { mobilität ohne eigentliche } \\
\text { Luxation }\end{array}$ & $\begin{array}{l}\text { Keine Traumaanamnese, generalisierte } \\
\text { ligamentäre Laxizität, Muskeldystrophie, } \\
\text { häufig bilateral }\end{array}$ \\
\hline Typ 2 & $\begin{array}{l}\text { Anterolaterale } \\
\text { Dislokation } \\
\text { (häufigster Typ) }\end{array}$ & $\begin{array}{l}\text { Verletzung der anterioren/ } \\
\text { posterioren Kapsel-Band- } \\
\text { Strukturen des PTFG, } \\
\text { häufig mit Läsion des } \\
\text { lateralen Kollateralbandes }\end{array}$ & $\begin{array}{l}\text { Sturz auf hyperflektiertes Knie mit } \\
\text { invertiertem/plantarflektiertem Fuß }\end{array}$ \\
\hline Typ 3 & $\begin{array}{l}\text { Posteromediale } \\
\text { Dislokation }\end{array}$ & $\begin{array}{l}\text { Zerreißen der Kapsel- } \\
\text { Band-Strukturen des PTFG } \\
\text { inkl. laterales Kollateral- } \\
\text { band }\end{array}$ & Direktes Trauma/Torsionsverletzung \\
\hline Typ 4 & $\begin{array}{l}\text { Kraniale } \\
\text { Dislokation } \\
\text { (selten) }\end{array}$ & $\begin{array}{l}\text { Assoziiert mit Zerreißen } \\
\text { der Membrana interossea } \\
\text { oder Tibiafraktur }\end{array}$ & $\begin{array}{l}\text { High-energy-OSG-Verletzung mit } \\
\text { Kranialisierung der gesamtem Fibula/ } \\
\text { bei kongenitaler Kniedislokation }\end{array}$ \\
\hline
\end{tabular}

Bei akuten Verletzungen sollte eine geschlossene Reposition in $80-110^{\circ}$ Flexion versucht werden. Die Notwendigkeit einer nachfolgenden Ruhigstellung für 36 Wochen wird kontrovers beurteilt $[1,5$, $7,8,9]]$. Bei erfolgloser geschlossener Reposition sowie posteromedialer/kranialer Dislokation muss diese direkt operativ mit gleichzeitiger Rekonstruktion der KapselBand-Strukturen und temporärer Fixation des PTFG erfolgen [7].

In der Literatur sind mehrere Möglichkeiten beschrieben, die von der Resektion des Fibulaköpfchens, über eine permanente/temporäre Arthrodese bis zur Rekonstruktion der Kapsel- und Bandstrukturen des PTFG mittels eines Anteils der BFS oder des Tractus iliotibialis reichen $[4,7,9,10]$.

Während bei der von Giachiano beschriebenen Technik die posteriore Hälfte der Bizepsfemorissehne durch die Tibia gezogen wird, mit zusätzlicher Augmentation durch einen Fascia-lata-Streifen [4], beschreibt Yanev eine Augmentation mit einem ventralen Sehnenstreifen der BFS [10]. Aufgrund des aus unserer Sicht im Bezug auf die Lage des tibialen Bohrlochs biomechanisch günstigeren Verlaufes verwendeten wir einen posterioren Sehnenstreifen, allerdings ohne zusätzliche Augmentation durch einen Fascia-lata-Streifen. Diese vereinfachte Technik führte in unserem Fall zu einem sehr guten Ergebnis mit in vollem Umfang beschwerdefrei sportfähigen Patienten.

Grenzen dieser Methode stellen sicher schon vorhandene arthrotische Verände- rungen des PTFG dar. Hier könnte wie durch Yanev beschrieben durch das Interponieren eines vaskularisierten $\mathrm{Fa}$ scia-lata-Streifens in das PTFG Abhilfe geschaffen werden [10]. Als Komplikationen bei ligamentärer Rekonstruktion werden Läsionen des N. peronaeus und eine Reinstabilität beschrieben. Nach Arthrodese des PTFG kommt es gehäuft zu Schmerzen und Instabilität im OSG, nach Resektion des Fibulaköpfchens zu Knieinstabilität und Schmerzen im OSG. Bei Kindern und Sportlern sind Arthrodesen/Resektionen des PTFG kontraindiziert $[3,5,7]$.

Aufgrund der Seltenheit dieser Pathologie gibt es in der Literatur bis auf einen 5-/9-Jahres-Follow-up [1] kaum Berichte über Langzeitverläufe nach operativer Therapie.

\section{Fazit für die Praxis}

Bei unklaren dorsolateralen Knieschmerzen muss klinisch und radiologisch gezielt nach einer chronischen Instabilität des PTFG gesucht werden, die leicht mit einer lateralen Meniskus-/Bandläsion verwechselt werden kann und im Verlauf zur sekundären Arthrose des PTFG führt. Nach primär konservativer Therapie sollte bei persistierenden Beschwerden nach 6 Monaten eine operative Therapie, z. B. durch Rekonstruktion der Kapsel-BandStrukturen mittels eines Anteils der Bizepsfemorissehne, in Betracht gezogen werden.

\section{Korrespondenzadresse Dr. M. Janke

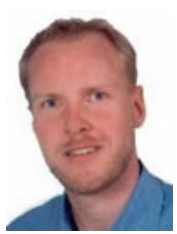 \\ Spital STS AG, Thun Krankenhausstrasse, 3600 Thun, Schweiz markus.janke@web.de}

Interessenkonflikt. Der korrespondierende Autor gibt an, dass kein Interessenkonflikt besteht.

\section{Literatur}

1. Aladin A et al. (2002) The importance of early diagnosis in the management of proximal tibiofibular dislocation: a 9-year follow-up of a bilateral case. Knee 9: 233-236

2. Esprengueira-Mendes JD et al. (2006) Anatomy of the proximal tibiofibular joint. Knee Surg Sport Traumatol Arthosc 14: 241-249

3. Falkenberg et al. (1983) Isolated anterior dislocation of proximal tibiofibular joint. J Bone Joint Surg Br 65: 310-311

4. Giachino AA (1986) Reccurent dislocation of the proximal tibiofibular joint: Report of two cases. J Bone Joint Surg Am 68: 1104-1106

5. Ogden JA et al. (1974) Subluxation and dislocation of the proximal tibiofibular joint. J Bone Joint Surg Am 56: 145-154

6. Ogden JA (1974) The anatomy and function of the proximal tibiofibular joint. Clin Orthop 101: 186191

7. Sekiya JK et al. (2003) Instability of the proximal tibiofibular joint. J Am Acad Orthop Surg 11: 120128

8. Semonian RH et al. (1955) Proximal tibiofibular subluxation relationship to lateral knee pain: review of proximal tibiofibular joint pathologies. J Orthop Sports Phys Ther21: 248-257

9. Van den Bekerom MP et al. (2004) Surgical stabilisation of the proximal tibiofibular joint using temporary fixation: a technical note. Acta Orthop Belg 70: 604-608

10. Yaniv M et al. (1999) A technical solution for secondary arthritis due to chronic proximal tibiofibular joint instability. Knee Surg Sport Traumatol Arthosc 7: 334-336

Hier steht eine Anzeige.

Dpringer 